\title{
Rare SNPs in receptor tyrosine kinases are negative outcome predictors in multiple myeloma
}

\author{
Sarah Keppler ${ }^{1,2, *}$, Susann Weißbach ${ }^{1,2,{ }^{*}}{ }$, Christian Langer ${ }^{3}$, Stefan Knop ${ }^{4}$, Jordan \\ Pischimarov ${ }^{1,2}$, Miriam Kull ${ }^{3}$, Thorsten Stühmer ${ }^{4}$, Torsten Steinbrunn ${ }^{4}$, Ralf \\ Bargou $^{2}$, Hermann Einsele ${ }^{4}$, Andreas Rosenwald ${ }^{1,2}$, Ellen Leich ${ }^{1,2}$ \\ ${ }^{1}$ Institute of Pathology, University of Würzburg, Würzburg, Germany \\ ${ }^{2}$ Comprehensive Cancer Center Mainfranken (CCC MF), University Hospital Würzburg, Würzburg, Germany \\ ${ }^{3}$ Department of Internal Medicine III, University Hospital UIm, UIm, Germany \\ ${ }^{4}$ Department of Internal Medicine II, University Hospital Würzburg, Würzburg, Germany \\ *These authors have equally contributed to this work
}

Correspondence to: Ellen Leich, email: Ellen.Leich@uni-wuerzburg.de

Keywords: multiple myeloma, receptor tyrosine kinases, amplicon sequencing, rare SNP

Received: January 20, 2016 Accepted: May 04, $2016 \quad$ Published: May 26, 2016

\section{ABSTRACT}

Multiple myeloma (MM) is a plasma cell disorder that is characterized by a great genetic heterogeneity. Recent next generation sequencing studies revealed an accumulation of tumor-associated mutations in receptor tyrosine kinases (RTKs) which may also contribute to the activation of survival pathways in MM. To investigate the clinical role of RTK-mutations in MM, we deep-sequenced the coding DNA-sequence of EGFR, EPHA2, ERBB3, IGF1R, NTRK1 and NTRK2 which were previously found to be mutated in MM, in 75 uniformly treated MM patients of the "Deutsche Studiengruppe Multiples Myelom". Subsequently, we correlated the detected mutations with common cytogenetic alterations and clinical parameters. We identified 11 novel nonsynonymous SNVs or rare patient-specific SNPs, not listed in the SNP databases 1000 genomes and dbSNP, in 10 primary MM cases. The mutations predominantly affected the tyrosine-kinase and ligand-binding domains and no correlation with cytogenetic parameters was found. Interestingly, however, patients with RTKmutations, specifically those with rare patient-specific SNPs, showed a significantly lower overall, event-free and progression-free survival. This indicates that RTK SNVS and rare patient-specific RTK SNPs are of prognostic relevance and suggests that MM patients with RTK-mutations could potentially profit from treatment with RTKinhibitors.

\section{INTRODUCTION}

Multiple myeloma (MM) is a malignant disease of plasma cells characterized by the accumulation of monoclonal plasma cells in the bone marrow $[1,2]$. The development proceeds via monoclonal gammopathy of undetermined significance and smoldering myeloma to symptomatic MM and plasma cell leukemia [3, 4]. On the molecular level, MM shows a great genetic heterogeneity with different clonal subgroups [2, 5]. Primary genetic events in the development of MM lead to the immortalization of differentiated plasma cells and include chromosomal translocations involving the $I G H$-locus on chromosome 14 such as $\mathrm{t}(4 ; 14), \mathrm{t}(11 ; 14), \mathrm{t}(14 ; 16), \mathrm{t}(8 ; 14)$ and hyperdiploidy $[3,4]$.

Moreover, the RAS/MAPK pathway, the JAK/STAT pathway and the PI3K/AKT pathway have previously been reported to be deregulated in $\mathrm{MM}$, leading to an increased proliferation and survival of MM cells [6-11]. In recent next generation sequencing studies single nucleotide variants (SNVs) have been observed in these pathways with $N R A S$ and $K R A S$ being among the most frequently mutated genes [5, 12-15]. Identical SNVs or single gene mutations, however, rarely occur in a significant amount of cases, but different SNVs do accumulate in specific signaling pathways. For example, we recently defined a signaling network composed of RTKs, adhesion molecules 
and their effectors and observed a tumor-associated SNV pattern that predicted inter- and intra-individual pathway redundancy [14]. RTKs are cell-surface receptors that have a conserved structure consisting of an extracellular region containing the ligand-binding domain, a transmembrane domain and an intracellular region containing the tyrosinekinase (TK) domain and additional regulatory regions [16]. Most RTKs are monomeric polypeptide chains in the absence of ligand-binding, with the exception of IGF1R which exists as a disulfide-linked dimer in the absence of a ligand $[17,18]$. RTKs dimerize upon ligand binding leading to autophosphorylation of the TK-domain and subsequent binding and activation of downstream effectors triggering signaling pathways including the PI3K/AKT and the RAS/ MAPK pathway subsequently leading to cell differentiation and proliferation [19-22]. However, while overexpression and mutations in the RTK FGFR3 have been shown in MM, [23-25] no information exists on how SNVs in other RTKs can effect MM development and progression. Given that RTKs play an important role in tumorigenesis and treatment of several cancer entities, [21, 26-29] we thus focused on the six RTK genes EGFR, EPHA2, ERBB3, IGF1R, $N T R K 1$ and NTRK2 that were previously described to be mutated in MM and deep-sequenced their coding DNA sequence (CDS) in biopsies of 75 primary MM cases of the "Deutsche Studiengruppe Multiples Myelom" (DSMM) taken at diagnosis.

While we focused on tumor-associated nonsynonymous SNVs in our previous whole exome sequencing study, we here investigated tumor-associated SNVs and non-synonymous SNPs before and after exclusion of SNPs listed in 1000 genomes and/or dbSNP. Specifically, we correlated the occurrence of SNVs, common SNPs and rare patient-specific SNPs with common cytogenetic alterations and/or clinical parameters to further elucidate their role in the clinical course of MM.

\section{RESULTS}

\section{Sequencing output, filtering and technical verification}

The CDS of EPHA2, ERBB3, IGF1R, NTRK1 and NTRK2 were covered on average with 2407, 2668, 2942, 2216 and 2370 reads/sample, respectively, and the CDS of EGFR, except for the ligand binding and TKdomain, with 2204 reads/sample. The ligand-binding and TK-domain of EGFR were sequenced with the 454 GS Junior and had an average coverage of 159 reads/sample (Supplementary Table S1, Supplementary Figure S1). The EGFR ligand-binding and TK-domain of one patient (P41) were not covered and therefore Sanger sequenced. Exons of EPHA2, NTRK1, IGF1R and EGFR with low coverage $(<10 \mathrm{x})$ were additionally sequenced by Sanger sequencing. However, no mutations were detected in these exons.

After first data processing, including read trimming, alignment and SNV calling, 156 mutations remained. 35 of the detected mutations were listed in the 1000 genome database and another 44 mutations in the dbSNPv134 and were excluded from the dataset. 26 mutations located in intronic regions, 2 mutations in untranslated regions, 1 mutation near a splice site and 18 synonymous mutations were removed. The remaining 30 mutations were verified by Sanger sequencing or high resolution melting assay (HRM) (Supplementary Figure S2). 9 mutations were only present in MM cell lines. All mutations that we previously detected in the 6 cell lines AMO1, INA6, JJN3, MM1.S, OPM2 and U266 by whole exome sequencing could also be detected in the current amplicon sequencing approach (Supplementary Table S2) [14]. 11 additional mutations that were detected by amplicon sequencing in primary MM cases could not be assessed by Sanger sequencing or high-resolutionmelting (HRM). Of those, 9 mutations had a low variant allele frequency (VAF) and far lower quality parameters than the mutations that could be technically verified, strongly suggesting false positive SNVs (Supplementary Table S3). One mutation was identified using the 454 GS Junior platform and included in the dataset, resulting in a total of 11 distinct heterozygous mutations in 10 primary MM cases (Table 1). All 11 mutations were not listed in 1000 genomes or dbSNP, were non-synonymous, located in conserved domains and led to structural changes in 4 out of 11 cases according to Polyphen2. 5 out of the 11 mutations $(45.5 \%)$ that occurred in $6 \mathrm{MM}$ cases with corresponding normal tissue available were also detected in the corresponding normal sample. Contamination problems could be excluded since the same DNA samples served as templates in a previously published study which did not reveal mutations in the respective normal samples [30]. Moreover, the mutation frequency in the corresponding normal sample was comparable to that in the tumor sample and patient P83 was affected by a germline mutation in the TK-domain of $E R B B 3$ but a somatic mutation in the ligand-binding domain of IGFIR (Figure 1B, 1D). To separate those patient-specific SNPs from the common SNPs listed in the databases 1000 genomes and dbSNP, we defined them as rare SNPs which also correlates to the information received from over 60000 individuals (allele frequency: $0-8.241 \times 10^{-5}$ ) using the ExAC browser (http://exac.broadinstitute.org/). 1 out of the 11 mutations (1/11 [9\%]) could be clearly defined as a tumor specific SNV and the remaining 5 mutations $(5 / 11[45 \%])$ could not be clearly assigned to one of the two groups due to the lack of corresponding normal tissue. They were specified as mutations-not otherwise specified (NOS). 
Table 1: Novel mutations detected in receptor tyrosine kinases

\begin{tabular}{lccccccccccccccccc}
\hline Gene & Chr. Exon & $\begin{array}{c}\text { Position } \\
\text { (hg19) }\end{array}$ & $\begin{array}{c}\text { Ref } \\
\text { Base }\end{array}$ & $\begin{array}{c}\text { Sample } \\
\text { Alleles }\end{array}$ & Patient & VAF & $\begin{array}{c}\text { cDNA } \\
\text { Pos. }\end{array}$ & AA & PolyPhen2 & PhastCons GERP \\
\hline EPHA2 & 1 & 6 & 16458908 & A & A/G & P82 & 49.75 & 2080 & TYR,HIS & benign & 0.824 & 4.95 \\
NTRK1 & 1 & 9 & 156841538 & A & A/C & P63 & 53.29 & 751 & ASN,HIS & probably-damaging & 1 & 4.97 \\
NTRK1 & 1 & 9 & 156841540 & C & C/G & P63 & 54.21 & 753 & ASN,LYS & probably-damaging & 0.998 & 1.05 \\
EGFR & 7 & 13 & 55229263 & G & G/C & P15 & 49.16 & 1570 & VAL,LEU & benign & 0.902 & 4.08 \\
EGFR & 7 & 27 & 55268938 & A & A/G & P56 & $42.86(F)$ & 3004 & MET,VAL & benign & 1 \\
& & & & & & & $35.42(\mathrm{R})$ & & & 5.65 \\
ERBB3 & 12 & 21 & 56491703 & G & G/T & P83 & 49.17 & 2595 & GLN,HIS & probably-damaging & 0.997 & 2.59 \\
ERBB3 & 12 & 23 & 56492567 & C & C/G & P31 & 24.38 & 2717 & THR,SER & benign & 0.999 \\
ERBB3 & 12 & 23 & 56492567 & C & C/G & P51 & 24.77 & 2717 & THR,SER & benign & 0.999 & 5.29 \\
IGF1R & 15 & 2 & 99251007 & C & C/T & P83 & 9.24 & 311 & THR,MET & probably-damaging & 0.998 & 5.36 \\
IGF1R & 15 & 18 & 99482518 & A & A/G & P40 & 26.43 & 3386 & ASN,SER & benign & 1 \\
IGF1R & 15 & 21 & 99500419 & G & G/T & P79 & 65.62 & 3852 & GLU,ASP & benign & 3.61 \\
IGF1R & 15 & 21 & 99500663 & A & A/C & P49 & 55.04 & 4096 & THR,PRO & benign & 0.576 \\
\hline
\end{tabular}

Chr. = Chromosome, $h \mathrm{~g}=$ human genome version 19, Ref Base $=$ reference base, $V A F=$ variant allele frequency, Pos. $=$ position, AA $=$ amino acid, $T Y R=$ tyrosine, HIS = histidine, $A S N=$ asparagine, $L Y S=$ lysine, $V A L=$ valine, $L E U=$ leucine, MET $=$ methionine, GLN $=$ glutamine, $T H R=$ threonine, $S E R=$ serine, $G L U=$ glutamic acid, $A S P=$ aspartic acid, $P R O=$ proline

\section{Accumulation of novel SNVs and rare SNPs in the kinase and ligand-binding domains of RTKs}

All 11 verified SNVs and rare SNPs detected by amplicon sequencing in the CDS of the RTKs EGFR, EPHA2, ERBB3, IGF1R, NTRK1 and NTRK2 were not described previously according to the cosmic database (http://cancer.sanger.ac.uk/cosmic) and they collectively affected 10 out of 75 primary MM cases $(13 \%)$ of the current study. Mutations that were previously detected in the cell lines L363, AMO1, MM1.S and U266 could be confirmed by the current amplicon approach (Figure 1A-1F) [14]. 4 rare SNPs and 2 mutations-NOS affected the TK-domains, 1 SNV and 2 mutations-NOS affected the ligand-binding domain, 1 rare SNP the furin-like domain and one mutation-NOS was found downstream of the TK-domain (Figure 1A-1F). The most affected RTK was $I G F 1 R$ which was mutated in 4 out of 75 patients (5.3\%), followed by ERBB3 (4\%), EGFR (2.7\%), EPHA2 and NTRK1 (each $1.3 \%$ ) as well as NTRK2 (0\%). More specifically, patients P82, P83/P51/P31 and P79 harbored a rare SNP in the TK-domain of EPHA2, ERBB3 and $I G F 1 R$, respectively (Figure 1B-1D). Interestingly in the case of ERBB3 P31 and P51 were affected by the same mutation, which in MM is a rarely-observed coincidence. Patients P56 and P40 were affected by a mutation-NOS in the TK-domain of EGFR and IGF1R, respectively (Figure 1A, 1D). Patient P83 had in addition a SNV in the ligandbinding domain of $I G F 1 R$ and patient P63 harbored 2 mutations-NOS in the immunoglobulin-like domain of
NTRK1 (Figure 1D, 1E), which is responsible for ligand binding in members of the NTRK family [31]. The furinlike domain of EGFR was affected by a rare SNP in patient $\mathrm{P} 15$ and a mutation-NOS was found downstream of the TK-domain of IGF1R (P49) (Figure 1A, 1D). A mutation in the CDS of NTRK2 was present in the MM cell line L363. However, no primary case was affected by SNVs, rare SNPs or mutations-NOS in NTRK2 (Figure $1 \mathrm{~F})$. Among the cell lines that were not included in the whole exome sequencing approach but investigated in the current amplicon sequencing approach, only RPMI8226 was affected by a mutation in 1 of the 6 RTKs, namely in the TK-domain of EGFR (Figure 1A). All detected SNVs and rare SNPs in primary MM cases and all mutations detected in MM cell lines are located in conserved regions as indicated by the GERP and PhastCons scores and 4 out of the 11 mutations have an influence on the protein structure according to the bioinformatics predictor PolyPhen2 (Table 1; Figure 1).

In summary, we detected 11 novel non-synonymous heterozygous mutations in 10 primary MM cases that were predominantly located in the kinase or ligand-binding domain of the six RTK genes analyzed.

\section{Rare patient-specific RTK SNPs are not restricted to hematopoietic tissue}

To answer the question whether the rare RTK SNPs are already present in the early phase of embryogenesis or may appear later during hematopoiesis, we investigated 


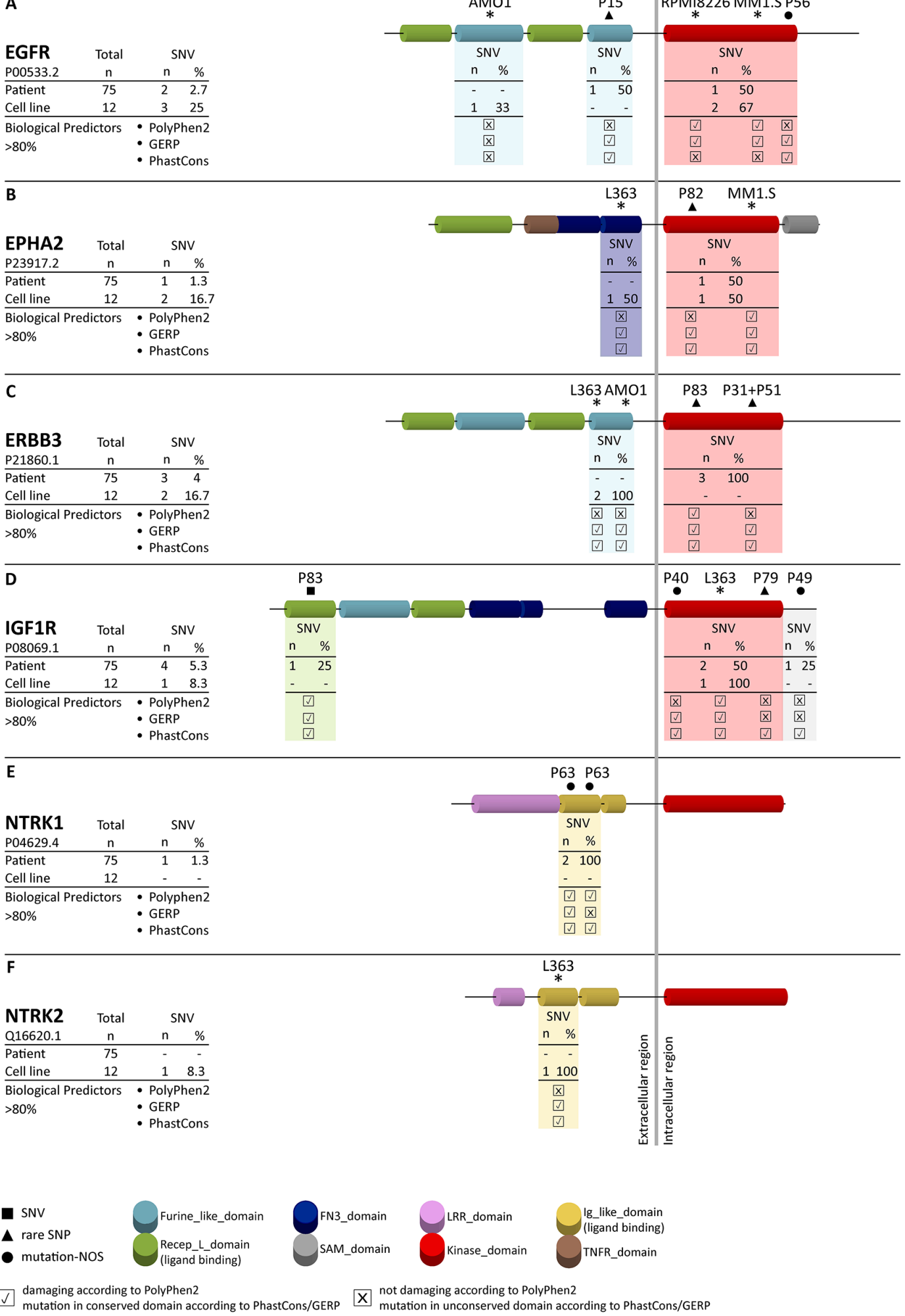

Figure 1: Frequency of mutations and affected regions in receptor tyrosine kinases. Amplicon sequencing revealed novel mutations in the receptor tyrosine kinases EGFR A. EPHA2 B. ERBB3 C. IGF1R D. NTRK1 E. and NTRK2 F. SNVs, rare SNPs and mutations-not otherwise specified (mutations-NOS) are indicated by a square $(\boldsymbol{\bullet})$, triangle $(\boldsymbol{\Delta})$ and circle $(\bullet)$, respectively, in combination with the corresponding patient number. Mutations in MM cell lines are indicated by asterisks. Functional predictions for each mutation in patients and MM cell lines are based on PhastCons and GERP (predicting the level of conservation) and PolyPhen2 (predicting structural changes). $\mathrm{x}$ indicates no influence of the mutation according to the functional predictor, $\checkmark$ indicates an influence of the mutation according to the functional predictor. 
non-hematopoietic paraffin embedded normal tissue of three patients (P51 [ERBB3], P83 [ERBB3], P79 $[I G F 1 R])$. We could confirm the ERBB3 SNP in the nonhematological normal samples of patients P51 (fatty skin of lower abdomen) and P83 (colon mucosa, liver, skin), as well as the IGF1R SNP in the non-hematological sample of patient P79 (colon) (Supplementary Figure S3). The occurrence of these SNPs in all non-hematological normal samples suggests that these mutations were inherited or acquired early in embryogenesis and did not arise later during hematopoiesis.

\section{RTK-mutations are not associated with DIS3 mutations and common cytogenetic events}

To further characterize the novel SNVs and rare SNPs detected in primary MM cases, we correlated all RTK-mutations with cytogenetic hallmarks that are commonly found in MM. Chromosomal translocations and genetic gains and losses were examined using FISH and the incidence of these cytogenetic events in our dataset was published previously (Supplementary Table S4) [30]. Mutations in the RTKs EGFR, EPHA2, ERBB3, $I G F 1 R$ and NTRK1 were not significantly associated with chromosomal losses of 13q14 and 17p13, chromosomal gains of 1q21, 9q34 and chromosomal translocations $\mathrm{t}(4 ; 14), \mathrm{t}(11 ; 14), \mathrm{t}(14 ; 16), \mathrm{t}(8 ; 14)$ and $\mathrm{t}(14 ; 20)$ (Table 2). Moreover, a correlation approach with mutated DIS3, one of the most frequently mutated genes in $\mathrm{MM}[12,13]$ revealed no significant association between mutations in DIS3 and mutations in the above mentioned RTKs (Table 2).

\section{RTK-mutations are adversely correlated with survival}

Although the 11 RTK-mutations did not correlate with bad prognostic factors such as deletions of $17 \mathrm{p} 13$, $13 q 14$ or $\mathrm{t}(4 ; 14)$ and were not associated with a worse response to therapy (Supplementary Table S5 [32]), we observed a significantly lower survival rate in RTK-mutant patients. Patients without a mutation $(n=62)$ had a median overall survival (OS) of 56 months, event-free survival (EFS) of 36 months and a progression-free survival (PFS) of 36 months while patients with a mutation $(n=8)$ had a median OS of 26 months, an EFS of 19 months and a PFS of 26 months, respectively ( $p=0.002, p=0.005, p=0.054$ ) (Figure 2A-2C).

To test whether the rare RTK SNPs alone have a significant impact on survival as well, we furthermore restricted our analysis to the MM patients that were only affected by rare germline mutations. Survival statistics revealed a significantly lower OS (56 months vs. 31 months, $p=0.011)$ and EFS (35 months vs. 21 months, $p=0.009$ ), and even a significantly lower PFS (36 months vs. 27 months, $p=0.025$ ) compared to the survival statistics that included all patients (Figure 3A-3C).
Due to the observation that the rare RTK SNPs that are not listed in dbSNPv134 and/or 1000 genomes have an influence on the survival of MM patients, we analyzed if common SNPs in general or common SNPs in conserved regions had an influence on survival as well. No significant differences in OS, EFS and PFS were observed in both analyses $((p=0.641, p=0.717$, $p=0.980)$ and $(p=0.667, p=0.516, p=0.786)$; Figure 4A$4 \mathrm{~F})$. In addition, we stepwise analyzed our detected SNPs reported in different dbSNP builds (up to build 134) to see if SNPs reported in higher builds rather than those reported in lower builds could also be associated with the survival of MM patients. Moreover, we investigated SNPs that occurred in a maximum of 2 of the 75 samples and may thus also fulfill the criteria of a rare SNP, separately. However, neither these stepwise analysis approaches $(n=12)$ nor the analysis of SNPs occurring in 2 samples or less showed a significant difference in survival of MM patients harboring SNPs of different builds, as compared to MM patients with no SNPs (OS: $p=0.184-0.848$, EFS: $p=0.215-0.793$, PFS: $p=0.254-0.952$; OS: $p=0.532$; EFS: $p=0.953$; PFS: $p=0.739$; respectively).

In summary, we could show that rare nonsynonymous MM-associated SNPs that are located in conserved domains of the RTKs EGFR, EPHA2, ERBB3, $I G F 1 R$ and NTRK1 have a significant influence on OS, EFS and PFS in patients of the current study cohort and might thus be of prognostic relevance.

\section{DISCUSSION}

Recent NGS studies revealed an accumulation of SNVs in RTKs, adhesion molecules and their downstream effectors and allowed to define a signaling network that was affected by at least one mutation in almost $100 \%$ of MM patients and more than one mutation in approximately $50 \%$ of MM patients [14].

This network also includes components of the MAPK and PI3K/AKT pathways, which can be activated by RTKs and have been reported to be consistently deregulated in MM [7, 19, 20, 33-35]. However, it is still a matter of debate as to whether recurrent mutations in key molecules of these pathways such as $R A S$ and $B R A F$ are of prognostic relevance. Depending on the study cohort and respective treatments, KRAS and NRAS mutations have adverse, beneficial or no effect on patient survival $[6,15,36-38]$. Mutations in $B R A F$ were found to improve the response to broad acting drugs, though the clinical impact of the V600E mutation is not clear yet [39, 40]. In addition, oncogenic activation of the PI3K/AKT pathway can only partially be explained by previous investigations $[11,41,42]$ and thus might also be explained by the occurrence of mutations in upstream molecules such as RTKs or other growth factors.

To further elucidate the role of RTK-mutations in MM, we deep-sequenced the CDS of the RTKs 
Table 2: Correlation of RTK mutations with cytogenetic events common in MM

\begin{tabular}{lccc}
\hline Cytogenetic Parameters & WT, $\mathbf{n}=\mathbf{6 5}$ & RTK_mut, $\mathbf{n}=\mathbf{1 0}$ & $\boldsymbol{p}$ \\
\hline 13q deletion; no, yes & $28 ; 37$ & $7 ; 3$ & 0.174 \\
17p deletion; no, yes & $53 ; 12$ & $8 ; 2$ & 1 \\
1q gain; no, yes & $42 ; 23$ & $6 ; 4$ & 1 \\
9q gain; no, yes & $38 ; 27$ & $3 ; 7$ & 0.17 \\
t(4;14); no, yes & $45 ; 20$ & $9 ; 1$ & 0.266 \\
t(11;14); no, yes & $50 ; 15$ & $7 ; 3$ & 0.695 \\
t(14;16); no, yes & $62 ; 3$ & $10 ; 0$ & 1 \\
t(8;14); no, yes & $62 ; 3$ & $9 ; 0$ & 1 \\
t(14;20); no, yes & $65 ; 0$ & $9 ; 0$ & - \\
DIS3 mut; no, yes & $56 ; 9$ & $10 ; 0$ & 0.598 \\
\hline
\end{tabular}

$W T=$ wild-type, $R T K=$ receptor-tyrosine kinase, mut $=$ mutated, $n=$ number, $p=p$-value

A
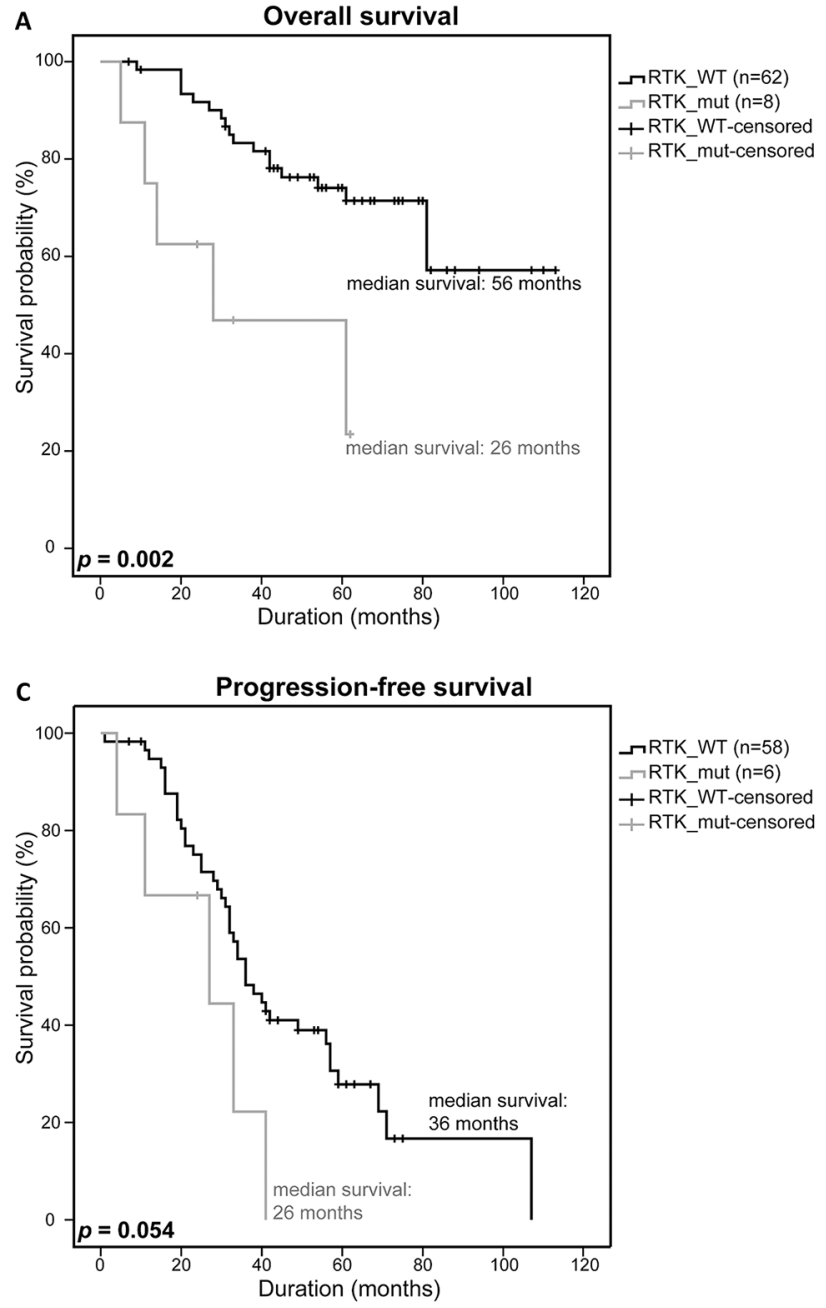

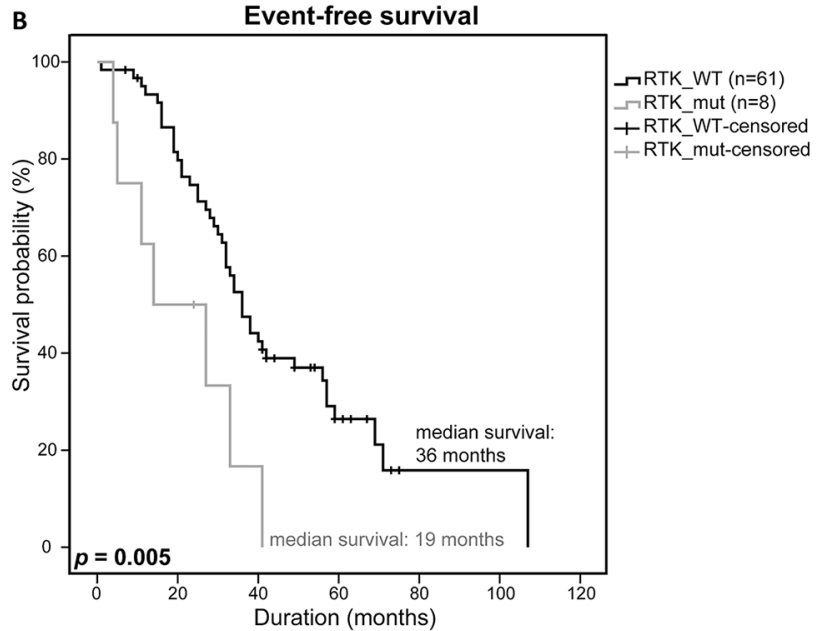

Figure 2: Clinical impact of RTK-mutations. Overall survival A. event-free survival B. and progression-free survival C. of patients with a RTK SNV, rare SNP or SNV/rare SNP (RTK_mut) were compared to patients with a WT-RTK profile (RTK_WT) using a univariate analysis with log-rank test for significance (OS: RTK_WT n=62, RTK_mut n=8; EFS: RTK_WT n=61, RTK_mut n=8; PFS: RTK_WT $\mathrm{n}=58$, RTK_mut $\mathrm{n}=6$ ). $P$-values $<0.05$ were considered statistically significant. 
EGFR, EPHA2, ERBB3, IGF1R, NTRK1 and NTRK2 that were found to be mutated in previous studies and investigated the association of these mutations with common cytogenetic alterations and clinical parameters in a study cohort of 75 patients of the DSMM that were uniformly treated with bortezomib, autologous stem cell transplantation and high dose chemotherapy.

Interestingly, 9 out of 11 non-synonymous SNVs and rare patient-specific SNPs that were detected in RTKs in the current approach and that mainly affected $I G F 1 R$ were located either in the TK- or ligand-binding domain and were not reported previously. Ligand binding leads to receptor dimerization, followed by auto-phosphorylation of the TK-domain and downstream signaling [19, 22]. Therefore, mutations in this domain could lead to discrepancies in ligand binding and subsequent downstream signaling. Additionally mutations in the TKdomain have been shown to have the potential to lead to constitutive phosphorylation and activation of RTKs
$[27,43]$. Both scenarios may trigger an adverse effect by a more sustained or accelerated downstream signaling promoting survival and proliferation of tumor cells and translating into a worse clinical outcome.

A significant association of RTK-mutations or rare RTK SNPs with common cytogenetic hallmarks of MM such as chromosomal gains of $1 \mathrm{q} 21$ or 9q34, chromosomal losses of $13 \mathrm{q} 14$ and $17 \mathrm{p} 13$, and the chromosomal translocations $\mathrm{t}(4 ; 14), \mathrm{t}(11 ; 14), \mathrm{t}(14 ; 16), \mathrm{t}(8 ; 14)$ and $\mathrm{t}(14 ; 20)$ was not observed (Table 2 and Supplementary Table S6).

Interestingly, however, our results - that need to be validated in a bigger patient cohort in a future study - strongly suggest that mutations in the RTKs have a significant impact on the survival of MM patients, specifically with regard to rare patient-specific SNPs (Figure 2, Figure 3). The occurrence of rare SNPs seem to be specific to the investigated RTKs based on the observation that such kind of rare SNPs were not detected
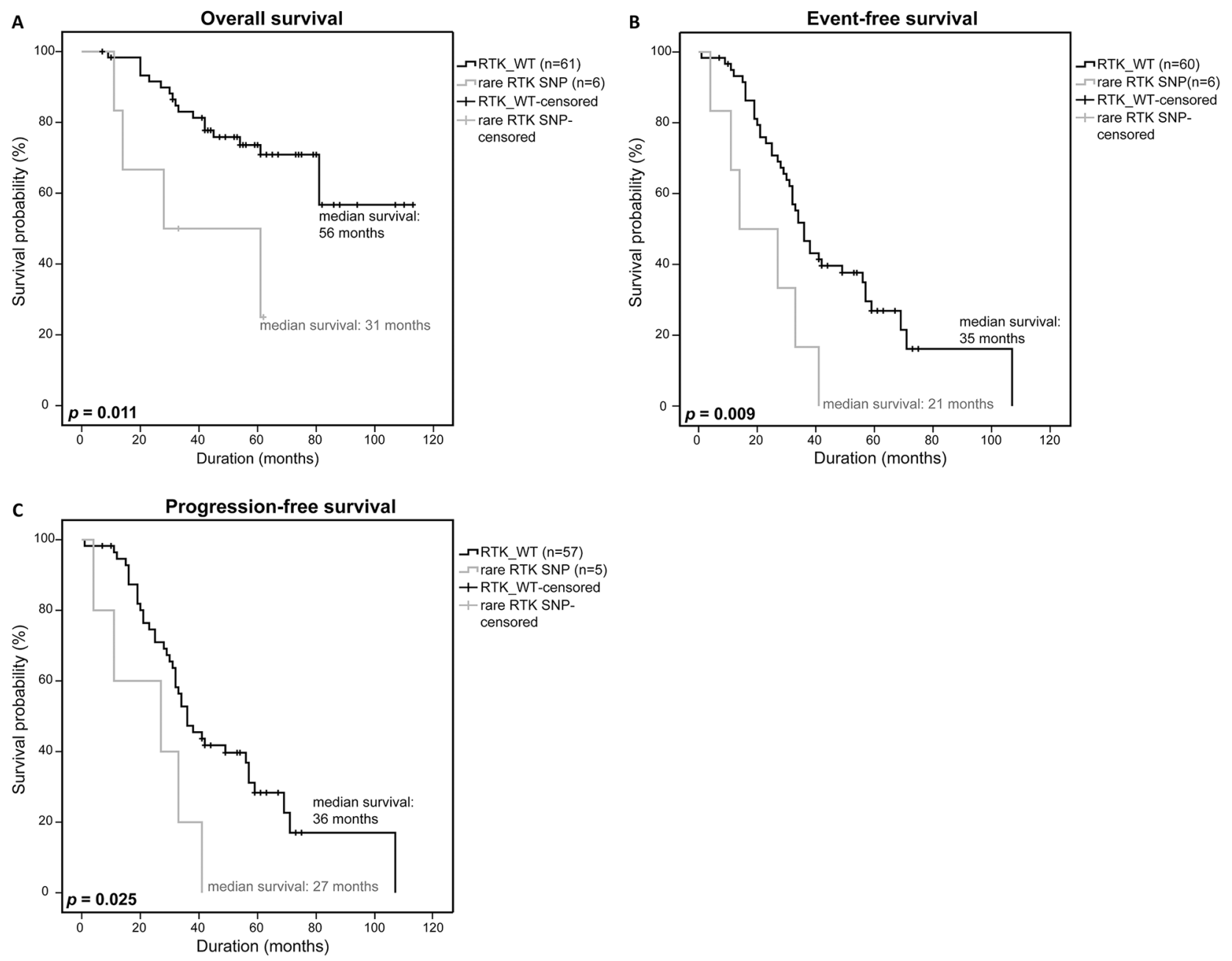

Figure 3: Clinical impact of rare RTK SNPs. Overall survival A. event-free survival B. and progression-free survival C. of patients with a rare RTK SNP were compared to patients without a RTK-mutation (RTK_WT) using a univariate analysis with log-rank test for significance (OS: RTK_WT n=61, rare RTK SNP n=6; EFS: RTK_WT n=60, rare RTK SNP n=6; PFS: RTK_WT n=57, rare RTK SNP $\mathrm{n}=7)$. $P$-values $<0.05$ were considered statistically significant. 
in DIS3 which was previously sequenced and analyzed in the same DSMM patient dataset [30]. The rare SNPs in RTKs that were identified in this study therefore seem to be non-random gene specific genetic changes that may serve as prognostic markers for MM patients.
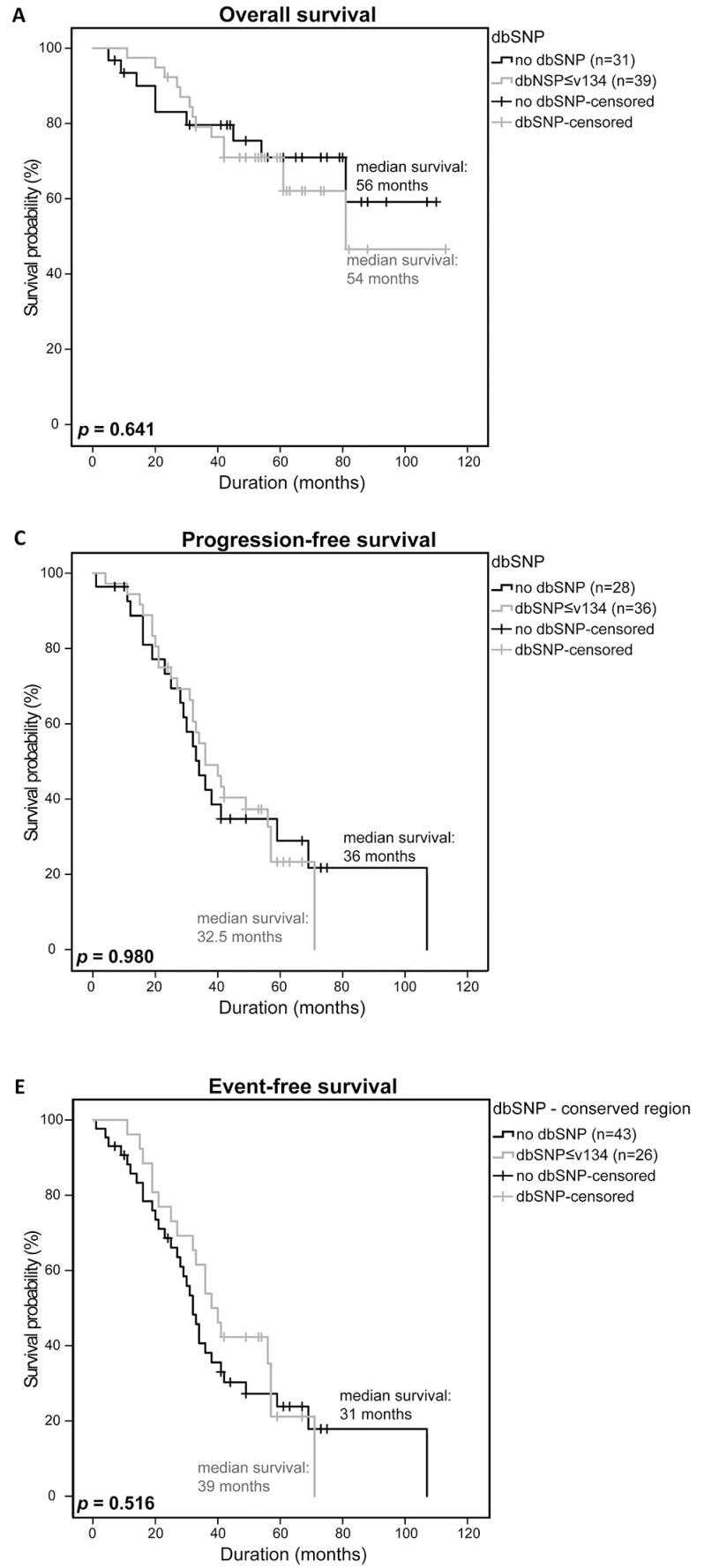

Since we previously showed that del17p13 and del13q14 are adverse prognostic factors in the current dataset, [30] we performed a correlation approach that disregarded cases with these deletions to test if it is really the RTK-mutations that account for the significant
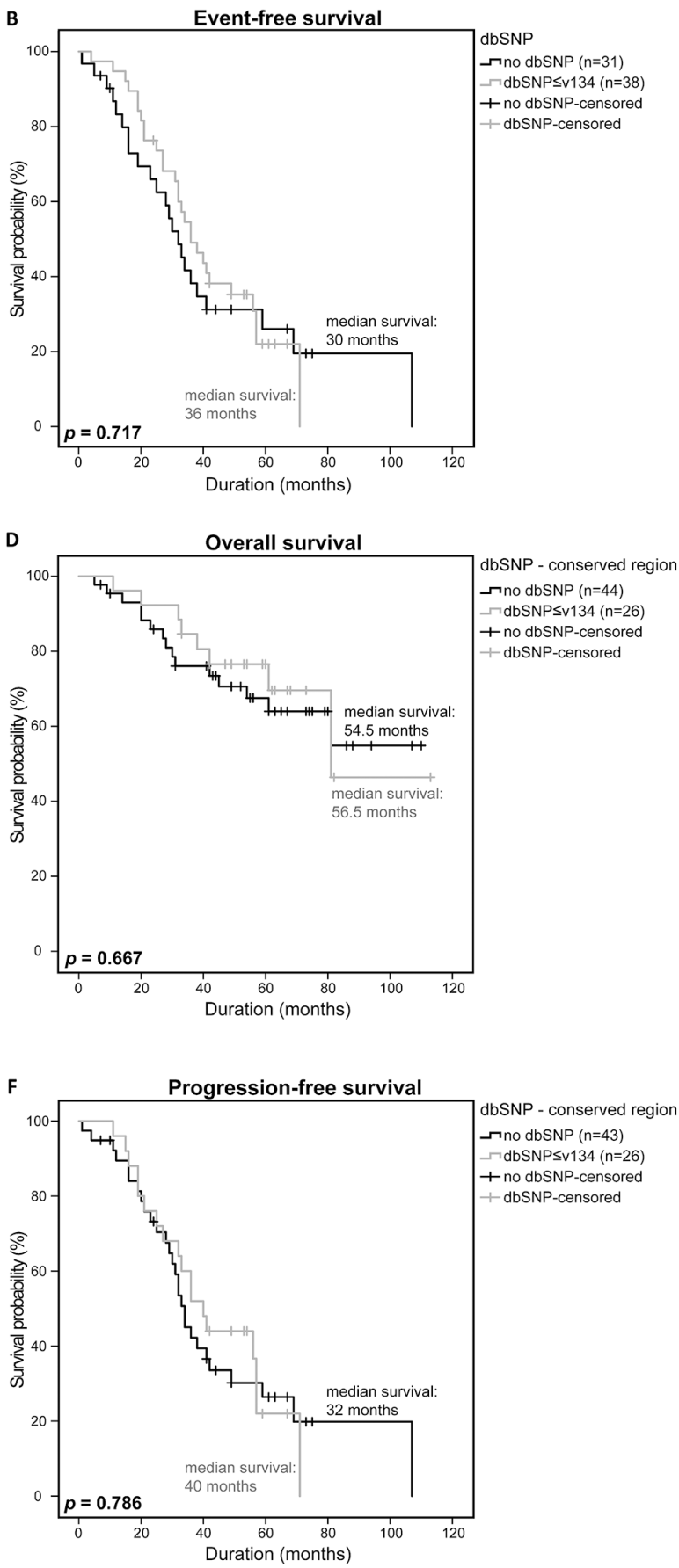

Figure 4: Clinical impact of RTK-mutations listed in dbSNP. Overall survival A. event-free survival B. and progression-free survival C. of patients with a general SNP listed in dbSNP $\leq$ v134 were compared to patients without a SNP using a univariate analysis with log-rank test for significance (OS: no dbSNP n=31, dbSNP n=39; EFS: no dbSNP n=31, dbSNP n=38; PFS: no dbSNP n=28, dbSNP n=36). Overall survival D. event-free survival E. and progression-free survival F. of patients with a SNP in a conserved region and listed in dbSNP $\leq \mathrm{v} 134$ were compared to patients without a SNP in a conserved region (OS: no dbSNP n=44, dbSNP n=26; EFS: no dbSNP n=43, dbSNP $\mathrm{n}=26$; PFS: no dbSNP n=43, dbSNP n=26). $P$-values $<0.05$ were considered statistically significant. 
differences in survival. Indeed, this analysis still revealed a significantly lower OS and EFS and a trend towards a lower PFS (del17p13: $p=0.003, p=0.009, p=0.118$, del13q14: $p=0.003, p=0.043, p=0.129$, respectively) (Supplementary Figure S4).

SNPs have been reported to influence cancer risk and progression in hematological and non-hematological malignancies, for example non-Hodgkin lymphoma, breast and prostate cancer [44-49]. However, only few genetic susceptibility factors have so far been described as risk factors for the development of $\mathrm{MM}$, namely SNPs in TERC(3q26.2), PSORS1C1(6p21.33), TNFRSF13B(17p11.2), $\quad C B X 7(22 \mathrm{q} 13.1), \quad$ DNMT3A (2p23.3), ULK4(3p22.1) and CDCA7L/DNAH11(7p15.3), as well as SNPs in $T N F \alpha$ and $L T \alpha$ [50-52].

If the rare RTK SNPs that we detected in the current analysis truly constitute predisposing risk factors in MM needs to be investigated in future studies. However, the presence of the rare RTK SNPs in non-hematopoietic tissue suggests that these mutations are either inherited or occur early in embryogenesis. This might indicate that rare RTK SNPs in MM are genetic events or predispositions that may act in concert with other mutations in MM rather than being initiators of tumorigenesis themselves.

To our knowledge, only the TNFa (-238) polymorphism has been described to be associated with improved survival in a study including $\mathrm{MM}$ patients treated with thalidomide [53] while there was only a trend towards an increased PFS in MM patients with the $T N F \alpha$ / $L T \alpha$ polymorphisms $(-308,+252)$ treated with high dose chemotherapy [52]. The rare RTK SNPs that we identified in the current study are thus the first reported SNPs that were significantly associated with a worse outcome in MM patients treated with bortezomib, autologous stem cell transplantation and chemotherapy.

The oncogenic role of aberrant RTK expression and function as well as the successful use of RTK inhibitors, for example in RTK-mutant patients with glioblastoma or colorectal cancer have been previously described [27-29]. Therefore, it might be useful to screen patients with MGUS or MM at diagnosis for the presence of RTKmutations, including rare patient-specific SNPs that may reveal potential susceptibilities to RTK-inhibitors.

\section{MATERIALS AND METHODS}

\section{Human multiple myeloma cell lines and primary multiple myeloma samples}

The study consisted of 75 primary MM samples from newly diagnosed symptomatic patients of the DSMM XI study and 12 MM cell lines. Primary samples were taken at initial diagnosis before treatment. Patients were treated with three cycles of combination therapy of bortezomib, dexamethasone and cyclophosphamide, subsequent stem cell mobilization, high-dose chemotherapy (HDC) and autologous stem cell therapy [54]. Clinical data was available for most patients (OS: $n=70$, EFS: $n=69$, PFS: $n=64$, response after bortezomib and HDC: $n=67$ and $\mathrm{n}=66$ ). MM cells were isolated using CD138 ${ }^{+}$microbeads (Miltenyi Biotec, Bergisch Gladbach, Germany) as described previously [55]. Corresponding normal samples were collected from either bone marrow aspirates, peripheral blood or paraffin embedded tissue. The study was approved by the Ethics Committee of the Medical Faculty, University of Würzburg (reference number: 18/09, approval renewed: 09.03.2009, reference number AZ 76/13, date of approval: 18.04.2013) and University of Ulm (application number: 307/08, date of approval: 21.01.2009). The clinical trial is registered under ClinicalTrials.gov number NCT00833560.

The human MM cell lines AMO1, JJN3, KMS11, KMS12BM, L363, MOLP8, NCIH929, OPM2, RPMI8226 and U266 were obtained from the German Collection of Microorganisms and Cell Cultures (DSMZ, Braunschweig, Germany). The cell line MM1.S was purchased from LGC Biolabs (Wesel, Germany). The INA6 cell line was a kind gift from Prof. Martin Gramatzki (Kiel, Germany). The MM cell lines were cultured in RPMI1640 medium (Life Technologies, Darmstadt, Germany) containing 10\% fetal bovine serum and 2mmol/1 L-glutamine (PAN Biotech, Aidenbach, Germany). INA6 cells were supplemented with $2 \mathrm{ng} / \mathrm{ml}$ human recombinant interleukin-6 and NCIH929 cells were supplemented with $0.05 \mathrm{mM}$ 2-mercapto-ethanol.

\section{Cytogenetic analysis}

Chromosomal translocations $\mathrm{t}(4 ; 14), \mathrm{t}(11 ; 14)$, $\mathrm{t}(14 ; 16), \mathrm{t}(8 ; 14)$ and $\mathrm{t}(14 ; 20)$, and genetic gains or losses of chromosome 1q21, 9q34, 13q14 and 17p13 were detected using fluorescence in situ hybridization (FISH) according to standard protocols $[56,57]$.

\section{Targeted resequencing of $E G F R, E P H A 2$, ERBB3, IGF1R, NTRK1 and NTRK2}

A library of the coding DNA sequences of $E G F R$, EPHA2, ERBB3, IGF1R, NTRK1 and NTRK2 was prepared using 50ng DNA from each sample. The DNA was purified using the AllPrep DNA/RNA Mini Kit (Qiagen, Hilden, Germany). Target regions were amplified in a multiplex PCR using 224 tagged primer pairs, designed by Fluidigm (Supplementary Table S7), with the 48.48 Access Array ${ }^{\mathrm{TM}}$ IFC and the FC1 Cycler System (Fluidigm, Amsterdam, The Netherlands) according to the manufacturer's protocol. Amplified target regions of each sample were pooled, diluted 1:100 and barcoded using the Access Array Barcode Library for Illumina Sequencers - 384 Single Direction (PN 100-4876, Fluidigm, Amsterdam, The Netherlands). Barcoded PCR products were analyzed on the 2100 Bioanalyzer (Agilent, Santa Clara, CA, USA), 
quantified using a Nanodrop spectrophotometer (Peqlab, Erlangen, Germany) and 20ng of each sample pool of one Access Array was pooled (Harvest sample pool). The Harvest sample pool was purified using AMPure-XP beads (BeckmanCoulter, Krefeld, Germany), analyzed on the 2100 Bioanalyzer and quantified using the Qubit system (Invitrogen, Darmstadt, Germany). An equal volume of each purified harvest sample pool of two performed Access Arrays was paired end sequenced on the MiSeq platform (Illumina, San Diego, USA) with a read length of $251 \mathrm{bp}$.

The library preparation and subsequent pyrosequencing of the coding sequences of the EGFR ligand-binding and TK-domain were performed on the 454 GS Junior (Roche, Mannheim, Germany) with an average read length of $351 \mathrm{bp}$ as described previously (primer information: Supplementary Table S8) [30].

Sequence data is deposited at the European Genomephenome Archive (EGA, http://www.ebi.ac.uk/ega/), hosted by the EBI (European Bioinformatics Institute), under accession number EGAS00001001665.

\section{Analysis of sequencing data}

FASTQ files generated on the MiSeq platform were trimmed using the Cutadapt 1.3 software to remove adaptor and barcode sequences (Supplementary Figure S2). Trimmed reads were aligned to the reference genome (hg19) using the BWA tool. Processing of the alignment result as well as detection, identification and quantitation of mutations were performed using the standard setting of the GATK software package. SeattleSeq-annotation 137 web-client was used to annotate SNVs to the reference genome hg19 to characterize SNVs (http://snp. gs.washington.edu/SeattleSeqAnnotation137/). Mutations that were already listed in the 1000 genomes (http:// www.1000genomes.org/) and the dbSNPv134 (http:// www.ncbi.nlm.nih.gov/SNP/) databases were excluded from the dataset. In a manual filter step we selected for non-synonymous mutations in coding regions. As part of the SeattleSeq137 annotation the bioinformatic predictors PhastCons $(-11.6-+5.82)$ and GERP (0-1) for conservation and PolyPhen2 (benign, probably damaging, possibly damaging) for structural changes were used to classify the presumed functional relevance of detected SNVs. Assuming that the lowest score predicts no relevance $(0 \%)$ and the highest score is equal to $100 \%$, we chose a threshold of $80 \%$ for GERP and PhastCons.

The sequencing data output from the 454 GS Junior was analyzed as described previously [30]. Results of both approaches were combined and used for further investigations (Supplementary Figure S2).

VAF of mutations of the MiSeq data were calculated manually, VAFs of the GS Roche Junior were calculated by the AVA software. For technical validation, sequencing results of six cell lines (AMO1, INA6, JJN3, MM1.S,
OPM2, U266) were compared with already existing whole-exome sequencing data (Supplementary Table S2) [14]. For technical verification, Sanger sequencing and HRM assays were performed. Unconfirmed mutations and mutations present only in cell lines were excluded from the statistical analysis. Mutations were manually assigned to protein domains using the NCBI protein database (http://www.ncbi.nlm.nih.gov/protein/).

\section{Sanger sequencing and high resolution melting assay}

Newly detected and already described SNVs with VAF $>20 \%$ were validated by Sanger sequencing in tumor and available corresponding normal sample according to standard protocols. Sequences were visualized using Chromas Lite (Technelysium, South Brisbane, Australia). SNVs with VAFs $<20 \%$ were validated using an HRM assay as described previously [30].

Primers for Sanger sequencing and HRM were purchased from Integrated DNA Technologies (Leuven, Belgium) and Eurofins Genomics (Ebersberg, Germany) (Supplementary Table S9).

\section{Statistical analysis}

Statistical analysis was performed using SPSS statistics software, version 23 (IBM, Ehningen, Germany). Kaplan-Meier curves were used for monovariate survival statistics in combination with log-rank tests for significance. Pearson chi-square and Fisher's exact-test were used for correlation of nominal variables. $P$-values $<$ 0.05 were considered statistically significant.

\section{ACKNOWLEDGMENTS}

We would like to thank Theodora Nedeva, Tina Grieb and Johanna Hammer for technical assistance.

\section{CONFLICTS OF INTEREST}

The authors declare no conflict of interest.

\section{GRANT SUPPORT}

This work was supported by the Deutsche Forschungsgemeinschaft - Clinical Research Unit 216 (KFO 216).

\section{REFERENCES}

1. Röllig C, Knop S and Bornhäuser M. Multiple myeloma. Lancet. 2015; 385: 2197-2208.

2. Egan JB, Shi CX, Tembe W, Christoforides A, Kurdoglu A, Sinari S, Middha S, Asmann Y, Schmidt J, Braggio E, 
Keats JJ, Fonseca R, Bergsagel PL, et al. Whole-genome sequencing of multiple myeloma from diagnosis to plasma cell leukemia reveals genomic initiating events, evolution, and clonal tides. Blood. 2012; 120: 1060-1066.

3. Morgan GJ, Walker BA and Davies FE. The genetic architecture of multiple myeloma. Nat Rev Cancer. 2012; 12: 335-348.

4. Prideaux SM, Conway O'Brien E and Chevassut TJ. The genetic architecture of multiple myeloma. Adv Hematol. 2014; 2014: 864058.

5. Walker BA, Wardell CP, Melchor L, Brioli A, Johnson DC, Kaiser MF, Mirabella F, Lopez-Corral L, Humphray S, Murray L, Ross M, Bentley D, Gutierrez NC, et al. Intraclonal heterogeneity is a critical early event in the development of myeloma and precedes the development of clinical symptoms. Leukemia. 2014; 28: 384-390.

6. Chng WJ, Gonzalez-Paz N, Price-Troska T, Jacobus S, Rajkumar SV, Oken MM, Kyle RA, Henderson KJ, Van Wier S, Greipp P, Van Ness B and Fonseca R. Clinical and biological significance of RAS mutations in multiple myeloma. Leukemia. 2008; 22: 2280-2284.

7. Bommert K, Bargou RC and Stühmer T. Signalling and survival pathways in multiple myeloma. Eur $\mathrm{J}$ Cancer. 2006; 42: 1574-1580.

8. Catlett-Falcone R, Landowski TH, Oshiro MM, Turkson J, Levitzki A, Savino R, Ciliberto G, Moscinski L, FernandezLuna JL, Nunez G, Dalton WS and Jove R. Constitutive activation of Stat 3 signaling confers resistance to apoptosis in human U266 myeloma cells. Immunity. 1999; 10: 105-115.

9. Hsu J, Shi Y, Krajewski S, Renner S, Fisher M, Reed JC, Franke TF and Lichtenstein A. The AKT kinase is activated in multiple myeloma tumor cells. Blood. 2001; 98: 2853-2855.

10. $\mathrm{Tu} \mathrm{Y}$, Gardner $\mathrm{A}$ and Lichtenstein A. The phosphatidylinositol 3-kinase/AKT kinase pathway in multiple myeloma plasma cells: roles in cytokine-dependent survival and proliferative responses. Cancer Res. 2000; 60: 6763-6770.

11. Steinbrunn T, Stühmer T, Gattenlöhner S, Rosenwald A, Mottok A, Unzicker C, Einsele H, Chatterjee M and Bargou RC. Mutated RAS and constitutively activated Akt delineate distinct oncogenic pathways, which independently contribute to multiple myeloma cell survival. Blood. 2011; 117: 1998-2004.

12. Lohr JG, Stojanov P, Carter SL, Cruz-Gordillo P, Lawrence MS, Auclair D, Sougnez C, Knoechel B, Gould J, Saksena G, Cibulskis K, McKenna A, Chapman MA, et al. Widespread genetic heterogeneity in multiple myeloma: implications for targeted therapy. Cancer Cell. 2014; 25: 91-101.

13. Chapman MA, Lawrence MS, Keats JJ, Cibulskis K, Sougnez C, Schinzel AC, Harview CL, Brunet JP, Ahmann GJ, Adli M, Anderson KC, Ardlie KG, Auclair $\mathrm{D}$, et al. Initial genome sequencing and analysis of multiple myeloma. Nature. 2011; 471: 467-472.
14. Leich E, Weißbach S, Klein HU, Grieb T, Pischimarov J, Stühmer T, Chatterjee M, Steinbrunn T, Langer C, Eilers M, Knop S, Einsele H, Bargou R, et al. Multiple myeloma is affected by multiple and heterogeneous somatic mutations in adhesion- and receptor tyrosine kinase signaling molecules. Blood Cancer J. 2013; 3: e102.

15. Walker BA, Boyle EM, Wardell CP, Murison A, Begum DB, Dahir NM, Proszek PZ, Johnson DC, Kaiser MF, Melchor L, Aronson LI, Scales M, Pawlyn C, et al. Mutational Spectrum, Copy Number Changes, and Outcome: Results of a Sequencing Study of Patients With Newly Diagnosed Myeloma. J Clin Oncol. 2015.

16. Hubbard SR. Structural analysis of receptor tyrosine kinases. Prog Biophys Mol Biol. 1999; 71: 343-358.

17. Ward CW, Lawrence MC, Streltsov VA, Adams TE and McKern NM. The insulin and EGF receptor structures: new insights into ligand-induced receptor activation. Trends Biochem Sci. 2007; 32: 129-137.

18. Adams TE, Epa VC, Garrett TP and Ward CW. Structure and function of the type 1 insulin-like growth factor receptor. Cell Mol Life Sci. 2000; 57: 1050-1093.

19. Lemmon MA and Schlessinger J. Cell signaling by receptor tyrosine kinases. Cell. 2010; 141: 1117-1134.

20. Schlessinger J. Cell signaling by receptor tyrosine kinases. Cell. 2000; 103: 211-225.

21. Blume-Jensen $P$ and Hunter T. Oncogenic kinase signalling. Nature. 2001; 411: 355-365.

22. Ullrich A and Schlessinger J. Signal transduction by receptors with tyrosine kinase activity. Cell. 1990; 61: 203-212.

23. Chesi M, Nardini E, Brents LA, Schrock E, Ried T, Kuehl WM and Bergsagel PL. Frequent translocation $\mathrm{t}(4 ; 14)$ (p16.3;q32.3) in multiple myeloma is associated with increased expression and activating mutations of fibroblast growth factor receptor 3. Nat Genet. 1997; 16: 260-264.

24. Intini D, Baldini L, Fabris S, Lombardi L, Ciceri G, Maiolo AT and Neri A. Analysis of FGFR3 gene mutations in multiple myeloma patients with $\mathrm{t}(4 ; 14)$. Br J Haematol. 2001; 114: 362-364.

25. Plowright EE, Li Z, Bergsagel PL, Chesi M, Barber DL, Branch DR, Hawley RG and Stewart AK. Ectopic expression of fibroblast growth factor receptor 3 promotes myeloma cell proliferation and prevents apoptosis. Blood. 2000; 95: 992-998.

26. Wilhelm SM, Carter C, Tang L, Wilkie D, McNabola A, Rong H, Chen C, Zhang X, Vincent P, McHugh M, Cao Y, Shujath J, Gawlak S, et al. BAY 43-9006 exhibits broad spectrum oral antitumor activity and targets the RAF/MEK/ERK pathway and receptor tyrosine kinases involved in tumor progression and angiogenesis. Cancer Res. 2004; 64: 7099-7109.

27. Krause DS and Van Etten RA. Tyrosine kinases as targets for cancer therapy. N Engl J Med. 2005; 353: 172-187.

28. Regad T. Targeting RTK Signaling Pathways in Cancer. Cancers (Basel). 2015; 7: 1758-1784. 
29. Hojjat-Farsangi M. Small-molecule inhibitors of the receptor tyrosine kinases: promising tools for targeted cancer therapies. Int J Mol Sci. 2014; 15: 13768-13801.

30. Weißbach S, Langer C, Puppe B, Nedeva T, Bach E, Kull M, Bargou R, Einsele H, Rosenwald A, Knop S and Leich E. The molecular spectrum and clinical impact of DIS3 mutations in multiple myeloma. Br J Haematol. 2015; 169: 57-70.

31. Holden PH, Asopa V, Robertson AG, Clarke AR, Tyler S, Bennett GS, Brain SD, Wilcock GK, Allen SJ, Smith SK and Dawbarn D. Immunoglobulin-like domains define the nerve growth factor binding site of the TrkA receptor. Nat Biotechnol. 1997; 15: 668-672.

32. Durie BG, Harousseau JL, Miguel JS, Blade J, Barlogie B, Anderson K, Gertz M, Dimopoulos M, Westin J, Sonneveld P, Ludwig H, Gahrton G, Beksac M, et al. International uniform response criteria for multiple myeloma. Leukemia. 2006; 20: 1467-1473.

33. Pratt RL and Kinch MS. Activation of the EphA2 tyrosine kinase stimulates the MAP/ERK kinase signaling cascade. Oncogene. 2002; 21: 7690-7699.

34. Pandey A, Lazar DF, Saltiel AR and Dixit VM. Activation of the Eck receptor protein tyrosine kinase stimulates phosphatidylinositol 3-kinase activity. J Biol Chem. 1994; 269: 30154-30157.

35. Zöllinger A, Stühmer T, Chatterjee M, Gattenlöhner S, Haralambieva E, Müller-Hermelink HK, Andrulis M, Greiner A, Wesemeier C, Rath JC, Einsele H and Bargou RC. Combined functional and molecular analysis of tumor cell signaling defines 2 distinct myeloma subgroups: Aktdependent and Akt-independent multiple myeloma. Blood. 2008; 112: 3403-3411.

36. Liu P, Leong T, Quam L, Billadeau D, Kay NE, Greipp P, Kyle RA, Oken MM and Van Ness B. Activating mutations of $\mathrm{N}$ - and $\mathrm{K}$-ras in multiple myeloma show different clinical associations: analysis of the Eastern Cooperative Oncology Group Phase III Trial. Blood. 1996; 88: 2699-2706.

37. Mulligan G, Lichter DI, Di Bacco A, Blakemore SJ, Berger A, Koenig E, Bernard H, Trepicchio W, Li B, Neuwirth R, Chattopadhyay N, Bolen JB, Dorner AJ, et al. Mutation of NRAS but not KRAS significantly reduces myeloma sensitivity to single-agent bortezomib therapy. Blood. 2014; 123: 632-639.

38. Gebauer N, Biersack H, Czerwinska AC, Schemme J, Hardel TT, Bernard V, Rades D, Lehnert H, Luley KB and Thorns C. Favorable prognostic impact of RAS mutation status in multiple myeloma treated with high-dose melphalan and autologous stem cell support in the era of novel agents: a single center perspective. Leuk Lymphoma. 2015: 1-4.

39. Andrulis M, Lehners N, Capper D, Penzel R, Heining C, Huellein J, Zenz T, von Deimling A, Schirmacher P, Ho AD, Goldschmidt H, Neben K and Raab MS. Targeting the BRAF V600E mutation in multiple myeloma. Cancer Discov. 2013; 3: 862-869.
40. Rustad EH, Dai HY, Hov H, Coward E, Beisvag V, Myklebost O, Hovig E, Nakken S, Vodak D, Meza-Zepeda LA, Sandvik AK, Wader KF, Misund K, et al. BRAF V600E mutation in early-stage multiple myeloma: good response to broad acting drugs and no relation to prognosis. Blood Cancer J. 2015; 5: e299.

41. Hofmann C, Stühmer T, Schmiedl N, Wetzker R, Mottok A, Rosenwald A, Langer C, Zovko J, Chatterjee M, Einsele H, Bargou RC and Steinbrunn T. PI3K-dependent multiple myeloma cell survival is mediated by the PIK3CA isoform. Br J Haematol. 2014; 166: 529-539.

42. Steinbrunn T, Stühmer T, Sayehli C, Chatterjee M, Einsele $\mathrm{H}$ and Bargou RC. Combined targeting of MEK/MAPK and $\mathrm{PI} 3 \mathrm{~K} /$ Akt signalling in multiple myeloma. Br J Haematol. 2012; 159: 430-440.

43. Paez JG, Janne PA, Lee JC, Tracy S, Greulich H, Gabriel S, Herman P, Kaye FJ, Lindeman N, Boggon TJ, Naoki K, Sasaki H, Fujii Y, et al. EGFR mutations in lung cancer: correlation with clinical response to gefitinib therapy. Science. 2004; 304: 1497-1500.

44. Loktionov A. Common gene polymorphisms, cancer progression and prognosis. Cancer Lett. 2004; 208: 1-33.

45. Charbonneau B, Wang AH, Maurer MJ, Asmann YW, Zent CS, Link BK, Ansell SM, Weiner GJ, Ozsan N, Feldman AL, Witzig TE, Cunningham JM, Dogan A, et al. CXCR5 polymorphisms in non-Hodgkin lymphoma risk and prognosis. Cancer Immunol Immunother. 2013; 62: 1475-1484.

46. Michailidou K, Hall P, Gonzalez-Neira A, Ghoussaini M, Dennis J, Milne RL, Schmidt MK, Chang-Claude J, Bojesen SE, Bolla MK, Wang Q, Dicks E, Lee A, et al. Large-scale genotyping identifies 41 new loci associated with breast cancer risk. Nat Genet. 2013; 45: 353-361, 361e351-352.

47. Eeles RA, Olama AA, Benlloch S, Saunders EJ, Leongamornlert DA, Tymrakiewicz M, Ghoussaini M, Luccarini C, Dennis J, Jugurnauth-Little S, Dadaev T, Neal DE, Hamdy FC, et al. Identification of 23 new prostate cancer susceptibility loci using the iCOGS custom genotyping array. Nat Genet. 2013; 45: 385-391, 391e1-2.

48. Zhang Y, Xia ZG, Zhu JH, Chen MB, Wang TM, Shen WX and He J. Association of Interleukin-10-3575T $>$ A and $-1082 \mathrm{~A}>\mathrm{G}$ polymorphisms with non-Hodgkin lymphoma susceptibility: a comprehensive review and meta-analysis. Mol Genet Genomics. 2015.

49. Baecklund F, Foo JN, Bracci P, Darabi H, Karlsson R, Hjalgrim H, Rosenquist R, Adami HO, Glimelius B, Melbye M, Conde L, Liu J, Humphreys K, et al. A comprehensive evaluation of the role of genetic variation in follicular lymphoma survival. BMC Med Genet. 2014; 15: 113.

50. Morgan GJ, Johnson DC, Weinhold N, Goldschmidt H, Landgren O, Lynch HT, Hemminki K and Houlston RS. Inherited genetic susceptibility to multiple myeloma. Leukemia. 2014; 28: 518-524.

51. Mitchell JS, Johnson DC, Litchfield K, Broderick P, Weinhold N, Davies FE, Gregory WA, Jackson GH, 
Kaiser M, Morgan GJ and Houlston RS. Implementation of genome-wide complex trait analysis to quantify the heritability in multiple myeloma. Sci Rep. 2015; 5: 12473.

52. Davies FE, Rollinson SJ, Rawstron AC, Roman E, Richards S, Drayson M, Child JA and Morgan GJ. High-producer haplotypes of tumor necrosis factor alpha and lymphotoxin alpha are associated with an increased risk of myeloma and have an improved progression-free survival after treatment. J Clin Oncol. 2000; 18: 2843-2851.

53. Neben K, Mytilineos J, Moehler TM, Preiss A, Kraemer A, Ho AD, Opelz G and Goldschmidt H. Polymorphisms of the tumor necrosis factor-alpha gene promoter predict for outcome after thalidomide therapy in relapsed and refractory multiple myeloma. Blood. 2002; 100: 2263-2265.

54. Kropff M, Liebisch P, Knop S, Weisel K, Wand H, Gann CN, Berdel WE and Einsele H. DSMM XI study: dose definition for intravenous cyclophosphamide in combination with bortezomib/dexamethasone for remission induction in patients with newly diagnosed myeloma. Ann Hematol. 2009; 88: 1125-1130.

55. Stühmer T, Arts J, Chatterjee M, Borawski J, Wolff A, King P, Einsele H, Leo E and Bargou RC. Preclinical anti-myeloma activity of the novel HDAC-inhibitor JNJ26481585. Br J Haematol. 2010; 149: 529-536.

56. Liebisch $\mathrm{P}$ and Dohner H. Cytogenetics and molecular cytogenetics in multiple myeloma. Eur J Cancer. 2006; 42: 1520-1529.

57. Ross FM, Avet-Loiseau H, Ameye G, Gutierrez NC, Liebisch P, O'Connor S, Dalva K, Fabris S, Testi AM, Jarosova M, Hodkinson C, Collin A, Kerndrup G, et al. Report from the European Myeloma Network on interphase FISH in multiple myeloma and related disorders. Haematologica. 2012; 97: 1272-1277. 